\title{
The cost-effectiveness of nCPAP treatment in patients with moderate-to-severe obstructive sleep apnoea
}

\author{
J. Mar*, J.R. Rueda\#, J. Durán-Cantolla ${ }^{\mp}$, C. Schechter ${ }^{+}$, J. Chilcott ${ }^{\S}$
}

The cost-effectiveness of $n C P A P$ treatment in patients with moderate-to-severe obstructive sleep apnoea. J. Mar, J.R. Rueda, J. Durán-Cantolla, C. Schechter, J. Chilcott. (C) ERS Journals Ltd 2003.

ABSTRACT: The demand for diagnostic and therapeutic services for obstructive sleep apnoea syndrome (OSAS) showed marked growth during the 1990s. This paper analyses the long-term cost-effectiveness of nasal continuous positive airway pressure (nCPAP) treatment in comparison to conventional null treatment.

A Markov model was used to represent the natural history of OSAS based upon published evidence. Utility values came from a survey of OSAS patients. Data on health costs were collected from hospitals in the Basque Country, Spain.

The incremental cost-effectiveness ratio of nCPAP treatment is $<6,000$ Euros per quality-adjusted life year. On disaggregated analysis, nCPAP treatment accounts for $86 \%$ of incremental costs; $84 \%$ of incremental effectiveness is attributable to improved quality of life.

Treatment of obstructive sleep apnoea syndrome with nasal continuous positive airway pressure has a cost-effectiveness that is in line with that of other commonly funded treatments such as antihypertensive drugs. The key clinical benefit of nasal continuous positive airway pressure treatment is improvement in the quality of life of patients with obstructive sleep apnoea syndrome. This benefit is also precisely the one for which the evidence base is strongest. The remaining uncertainties concerning the impact of nasal continuous positive airway pressure on long-term mortality have only a relatively small impact on the economics of treatment.

Eur Respir J 2003; 21: 515-522.
*Clinical Management Service, "Alto Deba" Hospital, Mondragón, ${ }^{\#}$ Dept of Preventive Medicine and Public Health, Basque Country University, Lejona, and Dept of Respiratory Medicine, Sleep Unit, Txagorritxu Hospital, VitoriaGasteiz, Spain. ${ }^{+}$Dept of Family Medicine \& Community Health, Albert Einstein College of Medicine, New York, NY, USA. ${ }^{8}$ School of Health and Related Research, University of Sheffield, Sheffield, UK.

Correspondence: J. Mar, Servicio de Gestión Clínica, Hospital "Alto Deba", 20500 Mondragón, Spain.

Fax: 34943035314

E-mail: jmar@hmon.osakidetza.net

Keywords: Cost-effectiveness analysis, Markov chains, obstructive sleep apnoea syndrome, quality of life

Received: May 172002

Accepted after revision: October 25 2002

This study was supported by the 2000 Commissioned Grant on sleep apnoea from the Basque Government Health Technology Agency (Vitoria, Spain) and Grant 01/0220 from the "Carlos III" Health Institute (Madrid, Spain).
Obstructive sleep apnoea syndrome (OSAS) [1] has been associated with increased cardiovascular morbidity and mortality [2-4], traffic accidents [5, 6] and a decline in quality of life [7-9]. Estimates of its prevalence in the adult population range $1-4 \%$ in males and $0.5-3 \%$ in females [10-12]. Both diagnosis, based on polysomnography, and treatment, with nasal continuous positive airway pressure (nCPAP), carry a potentially high cost [13]. Reflecting the potentially high prevalence, the demand for diagnostic and therapeutic services for OSAS has grown markedly during the 1990s. These two factors, high cost and growing demand, have led to OSAS being defined as a priority disease area for health technology assessment [14, 15]. Prominence has been given to the controversial review of WRIGHT et al. [15] questioning the available evidence about the effects of OSAS on cardiovascular morbidity and mortality and the benefit of treatment with nCPAP. Other authors have refuted these arguments, indicating the importance of analysing subgroups defined by severity of OSAS-related functional impairment and acceptance of the improvement in quality of life and risk of traffic accidents that treatment with CPAP provides to patients with severe OSAS [16-20].

Cost-effectiveness studies permit comparison of the benefits and costs of different treatments [21] and have been applied to
OSAS [7, 13, 22]. These studies focused on various diagnostic approaches to OSAS patients. They assume, implicitly, that nCPAP is an efficient treatment compared to other clinical practices and focus analysis on the different diagnostic strategies. Nevertheless, that assessment has not been proved in the literature. There is a lack of information about the impact on effectiveness and cost of introduction of nCPAP in the management of OSAS patients compared to the previous approach based on hygienic measures and follow-up. OSAS knowledge expanded during the 1980s [23]. It is time to determine whether treating it is comparable in cost-effectiveness to widely accepted treatments of other illnesses.

The present article presents an assessment of the costeffectiveness of nCPAP treatment in patients with OSAS and investigates the relative impact of the potential clinical benefits of treatment.

\section{Methods}

\section{Disease treatment pathway model}

Each individual OSAS patient lives out a lifetime that consists of moving through different health states over time 


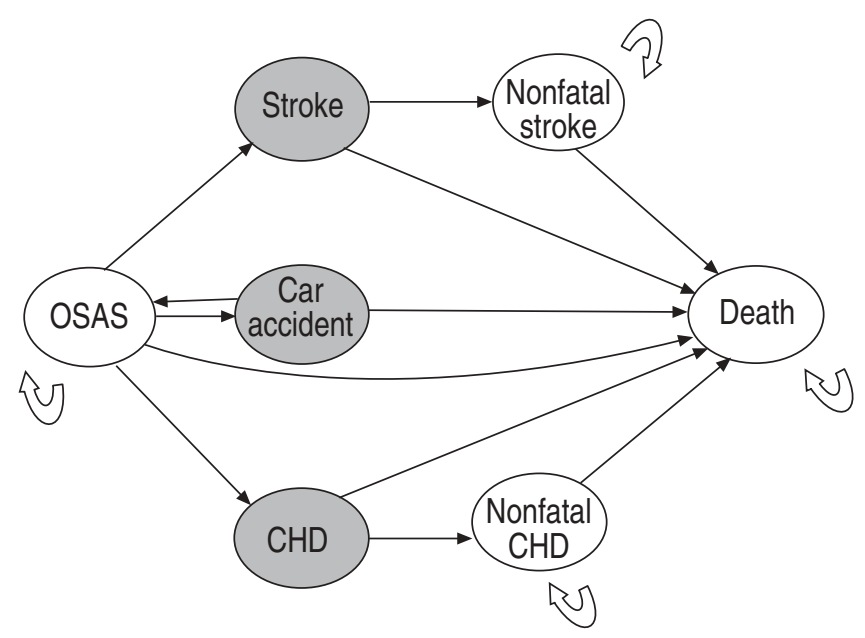

Fig. 1.- Natural history of obstructive sleep apnoea syndrome (OSAS) represented in a Markov-state diagram. Open arrows represent remaining in the same state and solid arrows transition between states ( $\square$ : Markov state; $\square$ : temporary event). CHD: coronary heart disease.

until death. Treatment with nCPAP changes both the length and quality of life that patients spend in each health state. A Markov model (semi-Markov) [24], implemented in DATA 3.5 (Tree-Age Software, Inc., Boston, MA, USA), was used to represent the natural history of OSAS (fig. 1) and calculate the mean time spent within each state. This figure shows the death risks that are increased in OSAS patients in comparison to the general population and that can be improved with nCPAP treatment: stroke, coronary heart disease (CHD), and car accidents. When the model starts running, all patients are in the state "OSAS". At the end of each cycle (1 yr), they must be in one of the following four states: "OSAS", "nonfatal stroke", "nonfatal CHD" and "death". During a cycle, patients can also experience a "car accident", "stroke" or "CHD" as temporary events. The "death" (absorbing) state is characterised by a zero probability of change. In a Markov model, the distribution of a population among various health states is calculated iteratively: the proportion in each health state at any given time is computed from the proportions in the health states during the preceding time period and known probabilities of changing from one health state to another. These probabilities are called transition probabilities and measure the probability of a patient moving from one state to another one during $1 \mathrm{yr}$. As the model runs, it calculates the time a patient spends in each state. Since the probabilities, costs and utilities are different, the model is run under the two alternative assumptions (table 1) to calculate the cost and effectiveness associated with each alternative together with the incremental or marginal cost-effectiveness of the novel strategy. The effectiveness measure employed is the quality-adjusted life year (QALY), described in the (Appendix); this measure has the advantage of combining the changes in mortality and morbidity into a single measure. The effectiveness of each alternative under analysis is calculated, by first, multiplying the quality-adjustment weight (utility) for each health state by the time spent in it and, secondly, summing the number of QALYs spent in each state.

The transition probabilities are derived from known epidemiological parameters such as age/sex-specific disease incidence and mortality rate, and vary over time. In the present model, they are based on mortality rates for stroke, CHD, traffic accidents and all causes, by age and sex, in the Basque Country, a region in the North of Spain with 2.3 million inhabitants. The numerator events used to calculate mortality rates for stroke include codes 430-438, and those for CHD codes 410-414, of the Clinical Modification of the ninth
Table 1. - Values of model parameters

\begin{tabular}{|c|c|c|c|}
\hline & $\begin{array}{c}\text { Untreated } \\
\text { OSAS }\end{array}$ & $\begin{array}{c}\text { Treated } \\
\text { OSAS }\end{array}$ & [Refs.] \\
\hline \multicolumn{4}{|l|}{ Probabilities ${ }^{\#}$} \\
\hline $\mathrm{CHD}$ & 1.185 & 1 & {$[2,25,26]$} \\
\hline Stroke & 1.353 & 1 & {$[2,25,26]$} \\
\hline Car accident & 8.1 & 1 & {$[5,20]$} \\
\hline Death after stroke & 1.1 & 1.1 & {$[26]$} \\
\hline Death after CHD & 1.1 & 1.1 & [26] \\
\hline \multicolumn{4}{|l|}{ Utilities } \\
\hline OSAS & 0.738 & 0.811 & Present study \\
\hline Nonfatal stroke & 0.590 & 0.649 & {$[27]$} \\
\hline Nonfatal CHD & 0.664 & 0.730 & [27] \\
\hline \multicolumn{4}{|l|}{ Costs $^{+}$} \\
\hline Diagnosis & 0 & 1028 & [28] \\
\hline Treatment with nCPAP & 0 & 358 & Present study \\
\hline Stroke hospitalisation & 3373 & 3373 & {$[26]$} \\
\hline CHD hospitalisation & 3128 & 3128 & [26] \\
\hline
\end{tabular}

OSAS: obstructive sleep apnoea syndrome; CHD: coronary heart disease; nCPAP: nasal continuous positive airway pressure. ${ }^{\#}$ : relative risk; ${ }^{\top}$ : European quality of life 5D scale; ${ }^{+}$: in Euros.

revision of the International Classification of Diseases. The mortality rates for traffic accidents include codes E810-E819. The transition probabilities from the "OSAS" state to the "death" state were based on mortality rates for all causes except stroke, CHD and car accidents. The Markov cycle duration was $1 \mathrm{yr}$. Transition probabilities were calculated as $\mathrm{p}=1-e^{- \text {rate }}$, assuming that the mortality rate is steady throughout each year. Half-cycle correction was applied to improve the accuracy of the results [24]. The general population mortality rates by age and sex were multiplied by the corresponding relative risks, shown in table 1 , to calculate the corrected rate applying to each subpopulation (treated and nontreated OSAS patients).

The parameters of the model, including relative risks, utilities and costs, are presented in table 1. The treatment strategy under analysis is the option of diagnosing OSAS and treating it using nCPAP. The conventional alternative consists of letting the natural progression of OSAS continue. Before the introduction of nCPAP, the natural history of OSAS patients could only be improved by treating other cardiovascular risk factors that were associated with it, such as hypertension, or conditions that aggravated its severity, such as obesity. These conditions are also treated when patients use nCPAP. Therefore, it is inappropriate to introduce interventions related to these other risk factors into the model because the measure calculated is the incremental cost-effectiveness ratio specifically related to nCPAP treatment and these interventions are introduced in both of the compared strategies. Effect on blood pressure was introduced because it was assumed that blood pressure returns to pre-OSAS levels as a consequence of the reduction in apnoea/hypopnoea index (AHI) by nCPAP, thereby improving stroke and CHD relative risks. Given uncertainty regarding this assumption [25], it was evaluated through sensitivity analysis, in which the benefit was allowed to range $100-25 \%$.

OSAS is a recognised risk factor for arterial hypertension [2], and relative risks for stroke and CHD [29] have been introduced into the model. The methodology and validation of this element of the model are presented in MAR and RodrigueZ-ARTALEJO [26]. The key assumption in assessment of cardiovascular risk is that, for OSAS patients with an AHI of $>30$, there is an increase in diastolic arterial pressure of $3.6 \mathrm{mmHg}$ [2]. The well-known meta-analysis of MACMAHON et al. [29] showed that increases in blood pressure are linearly correlated with the natural logarithm of the risk of stroke and 
CHD. By applying this relationship to the $3.6 \mathrm{mmHg}$ increase in diastolic pressure, the natural logarithms of the risks were obtained in order to calculate relative risks for both diseases. As is usual in cardiovascular epidemiology, it was assumed that the relative risk for stroke and CHD remain constant at a given blood pressure [30] and that absolute risk is best estimated by applying this relative risk to the baseline absolute risk for patient age and sex. This condition is introduced in the model by means of rates tables.

The base case is defined as a 50 -yr-old male patient with moderate-to-severe OSAS, defined by an AHI of $>30$ and daytime sleepiness (Epworth scale score of $>10$ ), typical of the patients served by the Sleep Unit of Txagorritxu Hospital, Vitoria-Gasteiz, Spain. The parameters (mean \pm SD) describing the last 5,000 patients referred for suspected OSAS are: $81 \%$ male, body mass index (BMI) $29.8 \pm 5.7 \mathrm{~kg} \cdot \mathrm{m}^{-2}$, patients with a BMI of $>30 \mathrm{~kg} \cdot \mathrm{m}^{-2} 51.2 \%$, AHI $25.7 \pm 25.3$, number of desaturations of $>3 \%$ per hour of sleep $24.5 \pm 22.0$, and percentage of sleep with an arterial oxygen saturation of $<90 \% 18.4 \pm 27.8$. Primary care physicians refer such patients to the Sleep Unit and, after clinical assessment, they undergo validated cardiorespiratory polygraphy, previously described elsewhere [31]. They are considered to have a low probability of OSAS if a respiratory disturbance index (RDI) of $<10$ according to cardiorespiratory polygraphy results. This occurs in over onethird of the patients $(36 \%)$, and another $29 \%$ are not considered candidates for treatment with nCPAP because they have only moderate or mild OSAS (RDI $<30$ and/or absence of symptoms). The remaining 35\% undergo split-time polysomnography to confirm the diagnosis of OSAS and for nCPAP titration. Of these, $10 \%$ abandon the treatment during the first year and, presumably, accrue no benefit. This equates to 3.2 patients undergoing diagnostic evaluation for every patient ultimately treated with nCPAP or a prevalence of $31.25 \%$ in the referred population.

Based on evidence regarding the long-term effectiveness of nCPAP [32, 33], the analytical time horizon used should be 5 yrs. However, sleep units are still treating patients who were diagnosed in the $1980 \mathrm{~s}$. In order to take both circumstances into account, the analysis was carried out using two time horizons: 5 yrs and the lifespan of the patient. Patient lifespan is the usual time horizon in the cost-effectiveness literature [21]. It was deemed preferable to calculate the cost-effectiveness of both extreme options rather than employ an intermediate value based upon arbitrary assumptions. Costs and health effects occurring in the future are discounted at 3\% per annum [21].

\section{Costs}

The cost analysis was undertaken from the perspective of the healthcare system. The direct costs of diagnosis and treatment of OSAS and costs attributable to cardiovascular morbidity were considered. The cost of diagnosis was estimated using data from the year 2000 and included the complete process followed by a patient with treated OSAS, as well as that of patients studied but not treated. The diagnostic protocol includes consultations with conventional testing (111 Euros $(€)$ ), cardiorespiratory polygraphy (€98) and polysomnography (€243). The diagnostic costs incurred in the population in order to treat one patient with nCPAP are $€ 1,028$. This figure includes the $10 \%$ drop-out rate from treatment. All costs were converted using exchange rates and prices current in 2000 .

The cost of treatment was calculated based on the price paid for the equipment in 2000 and the cost of its maintenance during the life of the patient. The annual cost $(€ 358)$ was calculated assuming a device life of 5 yrs and includes followup costs. The reference device was the CPAP S VI Plus
(ResMed, Sydney, Australia). Its price in 2001, with all supplies for $5 \mathrm{yrs}$, was $€ 1,256$. Assuming that its residual value after 5 yrs is null, the annual cost is $€ 251$. Other costs associated with nCPAP treatment are in-home technical maintenance twice a year $(€ 82)$ and medical follow-up once a year $(€ 25)$. Costs of treatment for cardiovascular events have been estimated using 1998 figures from all public hospitals in the Basque Country, adjusted to prices in 2000.

Patients with OSAS consume more healthcare services than the general population [34]. However, the effect of CPAP on this has not been measured; therefore, it was decided not to include it in the model.

\section{Utilities}

A survey was undertaken to obtain utility values using the European quality of life (EuroQoL) 5D instrument $[35,36]$. EuroQuol 5D is a preference-based health status and multiattribute utility scale that produces a single index score for each state of health. This score ranges $0-1$, where 1 is equivalent to full health and 0 to death. EuroQuol 5D was employed, although it is a generic questionnaire and other instruments, such as Functional Outcomes of Sleep Questionnaire [37] and the Epworth Sleepiness Scale [38], are specifically adapted to measure changes in quality of life in OSAS patients. The reason for this was that, in EuroQuol 5D, the weights used to score utilities were obtained using a preference elicitation technique such as time trade-off [36]. The OSAS-specific instruments are not adapted to economic evaluation because they are not preference-based and cannot be made to correspond to utilities. EuroQuol 5D and the Health Utilities Index [39], developed in Europe and Canada respectively, are highly respected examples of preferencebased utility scales. EuroQuol 5D was selected because it has been validated in the Spanish population.

The literature regarding utility values in OSAS patients reveals widely varying figures. The mean utility gain obtained using nCPAP ranges $0.24-0.05[7,9]$. Consequently, the authors decided to obtain their own data. Patients were recruited consecutively from the Sleep Unit of Txagorritxu Hospital and interviewed twice. The first interview was carried out before the beginning of treatment, and the second when the patient had used nCPAP for 3 months. There was no control group because the aim was to assess usual clinical practice in sleep units, which is focused on treatment of severe OSAS patients. Use of a control group would have required excluding the most severe cases. At the current level of knowledge of OSAS, it would have been unethical to delay treatment for 3 months.

The quality of life of patients with stroke and CHD is worse than that of the general population, but there is no information regarding quality of life in OSAS patients with stroke and CHD. This was modelled by assigning to these states qualityadjustment factors of 0.8 and 0.9 respectively [27] in relation to standard OSAS patient utilities.

\section{Sensitivity analysis}

The impact of age at diagnosis (30-60 yrs) and sex on the cost-effectiveness of treatment was explored. The impact of the improvements in quality of life due to nCPAP estimated by TousignanT et al. [7] and in the Trent study [40] is presented. In these analyses, the pretreatment patient utility value was used throughout and the utility gain, or effect size, of the treatment [41] was allowed to vary because the main driving force in the economic assessment is the difference 
Table 2. - Cost, effectiveness and incremental cost-effectiveness ratio (ICER) for the base case and two time horizons in males

\begin{tabular}{lccccc}
\hline Horizon & \multicolumn{2}{c}{ Cost $€$} & & \multicolumn{2}{c}{ Effectiveness QALY } \\
\cline { 2 - 4 } & No treatment & nCPAP & No treatment & nCPAP \\
\hline 5 yrs & 55 & 2719 & 3.39 & 3.73 & 7861 \\
Lifespan & 591 & 7902 & 12.90 & 14.38 & 4938 \\
\hline
\end{tabular}

Data are means. €: Euro; QALY: quality-adjusted life year; nCPAP: nasal continuous positive airway pressure.

between the values before and after treatment. The range tested was the $95 \%$ confidence interval of each survey utility gain.

It has been suggested that, in addition to hypertension, the risk of stroke could be related to independent factors [42]. Accordingly, a further analysis was carried out, in which it was assumed that these factors have the same impact as hypertension.

Another sensitivity analysis was performed to explore the impact of different diagnostic approaches. The diagnostic cost was adjusted as a function of OSAS prevalence and diagnostic protocol applied since these parameters determine cost directly. In this new scenario, the prevalence in referred patients was halved $(16 \%)$ and a more expensive diagnostic protocol introduced. In the second protocol, cardiorespiratory polygraphy (€98) was substituted for polysomnography (€243) in the initial screening and the second polysomnographic procedure was maintained for nCPAP titration. The consequence is an increase in diagnostic cost from $€ 1,028$ to $€ 2,700$, with intermediate diagnostic cost when only one parameter is changed.

The discount rate (1-5\%), proportion of patients who drop out from treatment $(10-50 \%)$ and nCPAP cost were also varied to complete the one-way sensitivity analysis. As, in some countries, nCPAP devices are not bought but rented at greater annual cost $(€ 622)$ [43], the analysis was repeated using this figure. An approach based on separate discounts for costs $(6 \%)$ and effectiveness $(1.5 \%)$ was also checked, as recommended by the National Institute for Clinical Excellence and UK Treasury (both London, UK) [40].

In order to measure the separate impact of the three therapeutic effects of nCPAP (disaggregated analysis) [21], additional analyses were performed for the baseline case, in which the effects of traffic accidents, cardiovascular risk and improvement in quality of life (utility) were evaluated separately. In order to calculate the incremental effectiveness of each therapeutic effect, the model was repeatedly computed, each time changing the relevant parameters in both alternatives (treatment and no treatment) and maintaining constant the parameters related to the other two effects. The treatment cost calculation was made computing the model only with nCPAP treatment cost and without taking into account stroke and CHD costs.

\section{Results}

Fifty-one patients completed the EuroQoL 5D questionnaire between October 1999-July 2000. One patient refused to take part in the study and four patients were not interviewed a second time. Thus, data were obtained from 46 patients, of whom $87 \%$ were male. The mean \pm sD age was $53 \pm 12 \mathrm{yrs}$, AHI $41.3 \pm 14.6$, Epworth score 13.8 \pm 5.8 and BMI $39.7 \pm 13.6$. The mean $(95 \%$ confidence interval) EuroQoL 5D score obtained was $0.738(0.646-0.829)$ before beginning nCPAP. The mean gain 3 months later was $0.073(0.015-0.131)$.

The incremental cost-effectiveness ratio for the base case is $€ 7,861 \cdot \mathrm{QALY}^{-1}$ if the time horizon is limited to $5 \mathrm{yrs}$ and $€ 4,938 \cdot$ QALY $^{-1}$ when the model is computed using the lifespan of the patient (table 2).
Table 3 shows the results of the sensitivity analysis by sex, age, treatment drop-out rate, discount rate, nCPAP cost, stroke relative risk and utility gain. The incremental costeffectiveness ratio is very stable and remains in the region $€ 5,000-10,000 \cdot \mathrm{QALY}^{-1}$ under these alternative assumptions. The results of the analysis are only shown by age and female sex. The other sensitivity analyses are limited to males because the results were similar for both sexes. The incremental costeffectiveness ratio of nCPAP treatment is only $>€ 20,000 \cdot \mathrm{QALY}^{-1}$ for the worst scenario evaluated (i.e. the lower limit of the authors survey's confidence interval and the 5-yr time horizon). The stroke relative risk, the discount rate, the treatment dropout rate and the beneficial effect of nCPAP on blood pressure have very little influence on the cost-effectiveness ratio. Conversely, the cost-effectiveness ratio clearly grows with nCPAP cost. As expected, separate analysis of increased cost and reduced

Table 3. - Sensitivity analysis by time horizon

\begin{tabular}{|c|c|c|c|}
\hline \multicolumn{2}{|c|}{ Sensitivity analysis } & \multicolumn{2}{|c|}{ ICER $€ \cdot \mathrm{QALY}^{-1}$} \\
\hline Parameter & Range tested & $5 \mathrm{yrs}$ & Lifespan \\
\hline \multicolumn{4}{|l|}{ Age yrs } \\
\hline Males & $30-60$ & 7896-7789 & $5035-4853$ \\
\hline Females & $30-60$ & 7879-7473 & $4734 \quad 4180$ \\
\hline RR stroke (untreated) & $1.353-1.706$ & $7861-7823$ & $4938-4634$ \\
\hline \multicolumn{4}{|l|}{ Utility gain } \\
\hline Present study & $0.013-0.126$ & $42235-4573 \quad 1$ & $17544-3021$ \\
\hline Ref. [7] & $0.112-0.367$ & $5142-1576$ & $3366-1092$ \\
\hline Ref. [36] & $0.09-0.16$ & $6388-3606$ & $4103-2418$ \\
\hline $\begin{array}{l}\text { Benefit of nCPAP } \\
\text { on blood } \\
\text { pressure } \%\end{array}$ & $100-25$ & 7861-7916 & $4938-5381$ \\
\hline Drop-out rate & $0.1-0.5$ & $7861-9381$ & $4938-5286$ \\
\hline Cost of nCPAP $€$ & $358-622$ & $7861-11444$ & $4938-8114$ \\
\hline Discount rate & $0.01-0.05$ & $7723-8001$ & $4603-4938$ \\
\hline Separate discount & $\begin{array}{c}\text { Cost 0.06; } \\
\text { effectiveness } 0.015\end{array}$ & 3653 & 3008 \\
\hline
\end{tabular}

ICER: incremental cost-effectiveness ratio; €: Euro; QALY: quality-adjusted life year; RR: relative risk; nCPAP: nasal continuous positive airway pressure.

Table 4.-Incremental cost-effectiveness ratio (ICER) of obstructive sleep apnoea syndrome (OSAS) treatment by time horizon and diagnostic scenario in population studied

\begin{tabular}{lcccc}
\hline OSAS & \multirow{2}{*}{$\begin{array}{c}\text { Diagnostic } \\
\text { prevalence }\end{array}$} & $\begin{array}{c}\text { Diagnostic } \\
\text { protocol }\end{array}$ & \multicolumn{2}{c}{ ICER $€ \cdot \mathrm{QALY}^{-1}$} \\
\cline { 4 - 5 } & & patient $€$ & $5 \mathrm{yrs}$ & Lifespan \\
\hline 0.32 & Present study & 1028 & 7861 & 4938 \\
0.16 & Present study & 1818 & 10193 & 5472 \\
0.32 & Two PSG & 1468 & 9160 & 5235 \\
0.16 & Two PSG & 2700 & 12796 & 6607 \\
\hline
\end{tabular}

$€$ : Euro; QALY: quality-adjusted life year; PSG: polysomnography. 
Table 5. - Dissaggregated incremental effectiveness in 50-yr-old males treated with nasal continuous positive airway pressure (nCPAP) for two time horizons

\begin{tabular}{|c|c|c|c|c|c|c|}
\hline & \multicolumn{2}{|c|}{ Car accidents } & \multicolumn{2}{|c|}{ Cardiovascular risk } & \multicolumn{2}{|c|}{ Utility effect } \\
\hline & Treated & Untreated & Treated & Untreated & Treated & Untreated \\
\hline RR car accidents & 1 & 8.1 & 1 & 1 & 1 & 1 \\
\hline RR CHD & 1 & 1 & 1 & 1.185 & 1 & 1 \\
\hline RR stroke & 1 & 1 & 1 & 1.353 & 1 & 1 \\
\hline Utility & 1 & 1 & 1 & 1 & 0.738 & 0.811 \\
\hline \multicolumn{7}{|l|}{ 5-yr time horizon } \\
\hline Effectiveness & 4.596 & 4.595 & 4.596 & 4.593 & 3.728 & 3.392 \\
\hline Incremental effectiveness ${ }^{\#}$ & $0.001(0.3)$ & & $0.003(0.9)$ & & $0.336(98.8)$ & \\
\hline \multicolumn{7}{|l|}{ Lifespan time horizon } \\
\hline Effectiveness & 17.729 & 17.691 & 17.729 & 17.513 & 14.378 & 13.084 \\
\hline Incremental effectiveness $\#$ & $0.038(2.4)$ & & $0.216(14.0)$ & & $1.294(83.6)$ & \\
\hline
\end{tabular}

Incremental effectiveness data are presented as disaggregated incremental effectiveness (percentage of total incremental effectiveness); RR: relative risk; CHD: coronary heart disease. ${ }^{\#}$ : difference between the effectiveness estimated with and without treatment for each scenario analysed.

effectiveness yields a decreased cost-effectiveness ratio. Table 4 shows the influence of diagnostic costs on cost-effectiveness. Tables 2-6 also show the relationship between cost-effectiveness and efficacy duration, expressed in terms of time horizon.

The relative contributions of the main factors affecting the incremental costs and effectiveness for the base case are shown in tables 5 and 6 . These results are very sensitive to time horizon. Over the whole life of the patient, improvements in quality of life account for $84 \%$ of the incremental effectiveness of nCPAP and the purchase and maintenance costs of nCPAP are responsible for $86 \%$ of the overall incremental cost. Reducing the time horizon to 5 yrs changes these figures to 98 and $61 \%$, respectively.

\section{Discussion}

There is no clear consensus in the health economics literature regarding the value of incremental cost-effectiveness ratio below which a treatment should be regarded as efficient. A controversial study, published in a Canadian context, stated that it lies in the range 20,000-100,000 Canadian dollars. QALY $^{-1}$ [44]. Tengs et al. [45], in a very extensive review of the cost-effectiveness of 500 life-saving interventions, found that the median cost per life year saved was US\$19,000 in the health sector. An assessment of the costeffectiveness of cardiovascular prevention programmes in Spain showed figures ranging US\$3,000->100,000 [46]. The present results are centred around $€ 5,000 \cdot \mathrm{QALY}^{-1}$ and only in the least favourable scenario rise above $€ 20,000 \cdot \mathrm{QALY}^{-1}$. Therefore, it can confidently be stated that treatment of OSAS with nCPAP has a cost-effectiveness in line with that of other widely used interventions.

In Txagorritxu Hospital Sleep Unit, following the recommendations of the Spanish Respiratory Society [47], patients with OSAS are treated when they have an AHI of $>30$ and exhibit symptoms of drowsiness. This decision was made taking into account the fact that nCPAP is not effective in patients with an AHI of $>30$ but without daytime sleepiness [48]. Consequently, the conclusions of the present study cannot be applied to less severe patients. In the literature on OSAS, definitions, epidemiology and natural history are considered together because there is a continuum between normality and severe OSAS [49]. In the same way, the current authors are in agreement with the comment of FLEETHAM [16] that understanding of the natural history of OSAS and nCPAP is at a stage similar to that of hypertension and hypercholesterolaemia in the 1970s. As with hypertensive patients, it is clear that severe patients should be treated, and the best recommendation for the mildest cases is simply monitoring. The problem is knowing what combination of AHI and symptomaticity separates these groups.

Obesity is a big health problem and its strong association with OSAS is likely to be causal [50]. It functions as a confounding factor in epidemiological studies measuring cardiovascular risk in OSAS patients. The multivariate models obtained in the Sleep Heart Health Study [51] suggest that the degree of obesity associated with the degree of sleepdisordered breathing, along with age and sex, explains most of the elevation in the other cardiovascular risk factors, with the exception of hypertension in males. Acknowledging this, obesity has been introduced into the present model only as a consequence of the blood pressure rise associated with an AHI of 30. The figures used were drawn from the populationbased study of YounG et al. [2] since it established the linear relationship between blood pressure and AHI adjusted for BMI. In the clinical approach to OSAS, obesity is a key factor that must be intensively assessed when present. In the costeffectiveness of OSAS treatment, the effect of obesity and its treatment on the natural history are excluded. OSAS can be a consequence of obesity but nCPAP does not produce weight changes. Conversely, if the cost-effectiveness of weight loss interventions were being estimated, OSAS should be introduced into the model to take into account their effect on sleep-disordered breathing.

As observed in table 5, the improvement in quality of life with nCPAP is the main driving force behind its clinical effectiveness measured in quality-adjusted life years. Its impact is seven times greater than that of reduced cardiovascular mortality, and this, in turn, is seven times greater than that of decreased numbers of traffic accidents. Other authors have reached the same conclusion; the available

Table 6. - Dissaggregated incremental cost in 50-yr-old males treated with nasal continuous positive airway pressure (nCPAP) for two time horizons

\begin{tabular}{|c|c|c|c|c|}
\hline \multirow[t]{2}{*}{ Horizon } & \multicolumn{2}{|c|}{ Diagnostic cost } & \multicolumn{2}{|c|}{ Treatment cost } \\
\hline & $€$ & $\%$ & $€$ & $\%$ \\
\hline $5 \mathrm{yrs}$ & 1028 & 39 & 1636 & 61 \\
\hline Lifespan & 1028 & 14 & 6238 & 86 \\
\hline
\end{tabular}

$€$ : Euro. 
evidence indicates that improved daytime alertness and quality of life represent the most important clinical end points of therapy since there is, as yet, insufficient evidence to justify prescribing nCPAP with the goal of reducing cardiovascular morbidity and mortality [52]. The present study has confirmed this assessment by quantifying the impact of each factor.

The utility values employed in the base case come from a survey performed using the EuroQoL 5D instrument. The difference in quality of life is similar to that obtained by JENKINSON et al. [9] using the EuroQoL 5D questionnaire (0.78-0.83). The improvement found by JENKINSON et al. [9] was much greater than that found employing other instruments, such as the 36-item short-form health survey and Patient-Generated Index. Similarly both TousignanT et al. [7] and the Trent study [40] found a larger improvement in quality of life before and after treatment, ranging 0.112-0.367 [7] and 0.09-0.16 [36], respectively. In spite of having used the most conservative estimates of effectiveness, the long-term incremental cost-effectiveness ratio remains $<€ 18,000 \cdot \mathrm{QALY}^{-1}$ in both males and females. From a clinical point of view, use of the EuroQoL 5D questionnaire could be seen as an odd decision because there are other questionnaires better adapted to OSAS. In the measurement of quality of life, the instrument used depends on the purpose and perspective of the study. The present study was designed to consider nCPAP in the context of overall resource allocation. The present authors would not employ the EuroQoL 5D questionnaire to assess the progression of the quality of life of an OSAS patient. Nevertheless, its property of being a preference-based health status scale and wide use help in the comparison of results with other treatment assessments [53].

The lack of a control group is a limitation of not only the present study but also all the literature regarding measurement of utility values in OSAS patients [7, 9, 40]. The increased utility calculated could be an overestimation since a placebo effect and regression to the mean could cause some of the improvement seen. Uncertainty about this parameter would be resolved if a study with a control group resolving ethical and technical constraints were carried out.

The relation of OSAS to car accidents has little effect on the cost-effectiveness of nCPAP treatment when analysis is undertaken, as here, at the population level. Consequently, a different approach should be used to assess groups prone to having car accidents, such as taxi drivers, or with great responsibility, such as coach drivers or aeroplane pilots.

Authors have paid much attention to different diagnostic approaches to OSAS [13, 14, 22, 28]. There are two elements that affect the efficiency of diagnosis, the prevalence of OSAS in the studied population and the diagnostic protocol employed. The cost of diagnosis per patient identified for nCPAP treatment increases as OSAS prevalence decreases. The diagnostic protocols employed depend on the use of polysomnography and cardiorespiratory polygraphy. In the least efficient scenario (table 5), prevalence is halved and polysomnography is used in the initial screening. Nevertheless, its impact on the economic efficiency of treatment of OSAS is quite low. In this context, the efficiency of use of nCPAP is reduced, although the costeffectiveness remains $>€ 7,000 \cdot \mathrm{QALY}^{-1}$. The reason for this is that the key factor in the overall cost is the cost of the nCPAP device.

It is well known that the cost of nCPAP differs greatly from one country to another, depending mainly on whether the device is bought or rented. Strictly speaking, what varies is not cost but charges. The cost is the aggregate value of the resources needed to provide a health or clinical intervention [21]. nCPAP device prices are similar across Europe. The main charge difference among countries appears because some National Health Services rent the devices, usually at higher charges. In
Spain, for instance, the nCPAP service to a patient is provided for $€ 622$ per year [43]. The decision regarding how to provide the service is made by policy makers. If that decision is not as efficient as it should be, responsibility for population-level nCPAP costs must not be assigned at the clinical level. The present authors are confident that the figure of $€ 358$ reflects the true cost of nCPAP treatment. There is no issue of statistical uncertainty in this parameter of the model.

Efficacy duration has quite an important impact on costeffectiveness ratios. The 5-yr time horizon is based on the available empirical evidence on efficacy duration but at the same time means that nCPAP becomes totally ineffective after that time. This assumption clearly contradicts general clinical practice in sleep units, which maintain the treatment beyond 5 or 10 yrs. Nonetheless, the decrease in efficiency, related to the 5-yr time horizon, does not change any of the main conclusions of the analysis. The cost-effectiveness ratios increase, but still remain within an efficient range.

In conclusion, treatment of obstructive sleep apnoea syndrome with nasal continuous positive airway pressure has a cost-effectiveness that is in line with that of other commonly funded healthcare interventions such as antihypertensive drugs and other cardiovascular risk factor treatments. There has been much controversy in the literature concerning the quality of the evidence regarding the link between treatment with nasal continuous positive airway pressure and cardiovascular mortality and road traffic accidents. This study puts that controversy into perspective: the key clinical benefit of nasal continuous positive airway pressure treatment is the improvement in the quality of life of patients with obstructive sleep apnoea syndrome. This is also precisely the benefit for which the evidence base is strongest. The remaining uncertainties concerning the impact of nasal continuous positive airway pressure on long-term mortality have only relatively little impact on the clinical and economic efficiency of treatment.

\section{Appendix: glossary}

The following definitions are taken from a report of the American Panel on Cost-Effectiveness in Health and Medicine [21].

Cost-effectiveness analysis. An analytical tool in which the costs and effects of a programme and at least one alternative are calculated and presented as the ratio of incremental cost to incremental effect.

Semi-Markov model. A type of Markov model that allows for systematic changes in transition probabilities as a function of simulated time rather than having constant transition probabilities. Also called time-varying Markov models.

Utility. A concept in economics, psychology and decision analysis referring to the preference for, or desirability of, a particular outcome.

Quality-adjusted life year. A measure of health outcome which assigns to each period of time a weight, ranging $0-1$, corresponding to the health-related quality of life during that period, where 1 corresponds to optimal health and 0 to a health state judged equivalent to death.

Discount rate. The interest rate used to compute present value or discount future sums.

\section{References}

1. Guilleminault C. Clinical features and evaluation of obstructive sleep apnea. In: Kryeger MH, Roth T, Dement WC, eds. Principles and Practice of Sleep Medicine. Philadelphia, PA, WB Saunders, 1989; pp. 552-558.

2. Young T, Peppard P, Palta M, et al. Population based study 
of sleep disordered breathing as a risk factor for hypertension. Arch Intern Med 1997; 157: 1746-1752.

3. Peppard PE, Young T, Palta M, Skatrud J. Prospective study of the association between sleep-disordered breathing and hypertension. $N$ Engl J Med 2000; 342: 1378-1384.

4. Lavie P, Herer P, Hoffstein V. Obstructive sleep apnoea syndrome as a risk factor for hypertension: population study. BMJ 2000; 32: 479-482.

5. Terán-Santos J, Jimenez-Gomez A, Cordero-Guevara J. The association between sleep apnea and the risk of traffic accidents. $N$ Engl J Med 1999; 340: 847-851.

6. Horstmann S, Hess CW, Bassetti C, Gugger M, Mathis J. Sleepiness-related accidents in sleep apnea patients. Sleep 2000; 23: 383-389.

7. Tousignant P, Cosio MG, Levy RD, Groome PA. Quality adjusted life years added by treatment of obstructive sleep apnea. Sleep 1994; 17: 52-60.

8. Engleman HM, Martin SE, Deary IJ, Douglas NJ. Effect of continuous positive airway pressure treatment on daytime function in sleep apnoea/hypopnoea syndrome. Lancet 1994; 343: $572-575$.

9. Jenkinson C, Stradling J, Petersen S. How should we evaluate health status? A comparison of three methods in patients presenting with obstructive sleep apnoea. Qual Life Res 1998; 7: 95-100.

10. Young T, Palta M, Dempsey J, Skatrud J, Webwe S, Badr S. The occurrence of sleep-disordered breathing among middleaged adults. $N$ Eng $J$ Med 1993; 328: 1230-1235.

11. Gibson GJ, Douglas NJ, Stradling JR, London DR, Semple SJ. Sleep apnoea: clinical importance and facilities for investigation and treatment in the UK. Addendum to the 1993 Royal College of Physicians Sleep Apnoea report. $J R$ Coll Physicians Lond 1998; 32: 540-544.

12. Durán J, Esnaola S, Rubio R, Iztueta A. Obstructive sleep apnea-hypopnea and related clinical features in a population-based sample of subjects aged 30 to 70 years. $\mathrm{Am}$ J Respir Crit Care Med 2001; 163: 1-5.

13. Chervin RD, Murman DL, Malow BA, Totten V. Costutility of three approaches to the diagnosis of sleep apnea: polysomnography, home testing and empirical therapy. Ann Intern Med 1999; 130: 496-505.

14. Pack AI, Gurubhagavatula I. Economic implications of the diagnosis of obstructive sleep apnea. Ann Intern Med 1999; 130: $533-534$.

15. Wright J, Johns R, Watt I, Melville A, Sheldon T. Health effects of obstructive sleep apnoea and the effectiveness of continuous positive airways pressure: a systematic review of the research evidence. BMJ 1997; 314: 851-860.

16. Fleetham JA. A wake up call for sleep disordered breathing. Evidence of ill effects is conflicting and inconclusive. $B M J$ 1997; 314: 839-840.

17. Stradling JR, Davies RJ. Obstructive sleep apnoea. Evidence for efficacy of continuous positive airways pressure is compelling. BMJ 1997; 315: 368.

18. Shneerson J, Smith I. Obstructive sleep apnoea. False impression of objectivity may deny patients affordable treatment. BMJ 1997; 315: 367.

19. Pack AI, Young T. Obstructive sleep apnoea. Superficial analysis ignores evidence on efficacy of treatment. $B M J$ 1997; 315: 367-368.

20. Cassel W, Ploch T, Becker C, Dugnus D, Peter JH, von Wichert P. Risk of traffic accidents in patients in sleepdisordered breathing: reduction with nasal CPAP. Eur Respir $J$ 1996; 9: 2606-2611.

21. Gold MR, Siegel JE, Russell LB, Weinstein MC. CostEffectiveness in Health and Medicine. New York, NY, Oxford University Press, 1996.

22. Reuveni H, Schweitzer E, Tarasiuk A. A cost-effectiveness analysis of alternative at-home or in-laboratory technologies for the diagnosis of obstructive sleep apnea syndrome. Med Decis Making 2001; 21: 451-458.

23. Lavie P. Nothing new under the moon: historical accounts of sleep apnea syndrome. Arch Intern Med 1984; 144: 20252028.

24. Sonnenberg FA, Beck R. Markov models in medical decision making: a practical guide. Med Decis Making 1993; 13: 322-338.

25. Pankow W, Lies A, Friedrich W. Sleep-disordered breathing and hypertension. $N$ Engl J Med 2000; 343: 966-967.

26. Mar J, Rodriguez-Artalejo F. Which is more important for the efficiency of hypertension treatment: hypertension stage, type of drug or therapeutic compliance? J Hypertens 2001; 19: $149-155$.

27. Torrance GW, Feeny D. Utilities and quality-adjusted life years. Int J Technol Assess Health Care 1989; 5: 559-575.

28. Chesson AL, Ferber RA, Fry JM, et al. The indications for polysomnography and related procedures. Sleep 1997; 20: 423-487.

29. MacMahon S, Peto R, Cutler J, et al. Blood pressure, stroke, and coronary heart disease. Part 1, prolonged differences in blood pressure: prospective observational studies corrected for the regression dilution bias. Lancet 1990; 335: 765-774.

30. Zanchetti A. Antihypertensive therapy: how to evaluate the benefits. Am J Cardiol 1997; 79: 3-8.

31. Esnaola S, Durán J, Infante-Rivard C, Rubio R, Fernández A. Diagnostic accuracy of a portable recording device (MESAM IV) in suspected obstructive sleep apnoea. Eur Respir J 1996; 9: 2597-2605.

32. Chaouat A, Weitzenblum E, Kessler R, et al. Five-year effects of nasal continuous positive airway pressure in obstructive sleep apnoea syndrome. Eur Respir J 1997; 10: 2578-2582.

33. Inoue Y, Nanba K, Hazama G, Takata K, Arai H. Longterm follow-up study on patients with sleep apnea syndrome. Psychiatry Clin Neurosci 2001; 55: 245-246.

34. Ohayon MM, Guilleminault C, Priest RG, Caulet M. Snoring and breathing pauses: results in the general population of the United Kingdom. BMJ 1997; 314: 860863.

35. EuroQoL Group. EuroQoL - A new facility for measurement of health-related quality of life. Health Policy 1990; 16: 199-208.

36. Dolan P, Gudex C, Kind P, Williams A. The time trade-off method: results from a general population study. Health Econ 1996; 5: 141-154.

37. Weaver TE, Laizner AM, Evans LK, et al. An instrument to measure functional status outcomes for disorders of excessive sleepiness. Sleep 1997; 20: 835-843.

38. Johns MW. Daytime sleepiness, snoring and obstructive sleep apnea. The Epworth Sleepiness Scale. Chest 1993; 103: 30-36.

39. Feeny D, Furlong W, Boyle M, Torrance GW. Multiattribute health status classification systems. Health Utilities Index. Pharmacoeconomics 1995; 7: 490-502.

40. Chilcott J, Clayton E, Chada N, Hanning CD, Kinnear W, Waterhouse JC. Nasal Continuous Positive Airways Pressure in the Management of Sleep Apnoea. Sheffield, Trent Institute for Health Services Research, Universities of Leicester, Nottingham and Sheffield, 2000.

41. Kazis LE, Anderson JJ, Meenan RF. Effect sizes for interpreting changes in health status. Med Care 1989; 27: Suppl., S178-S189.

42. Mohsenin V. Sleep-related breathing disorders and risk of stroke. Stroke 2001; 32: 1271-1278.

43. Mar J, Rueda JR, Duran J. Análisis coste-efectividad de los tratamientos del síndrome de la apnea del sueño en la Comunidad Autónoma del País Vasco. Informe $n^{\circ}$ : Osteba D-01-01. Vitoria-Gasteiz, Departamento de Sanidad, Gobierno Vasco, 2000.

44. Laupacis A, Feeny D, Detsky AS, Tugwell PX. How attractive does a new technology have to be to warrant adoption and utilization? Tentative guidelines for using clinical and economic evaluation. Can Med Assoc J 1992; 146: 473-481.

45. Tengs OT, Adams ME, Pliskin JS, et al. Five-hundred 
life-saving interventions and their cost-effectiveness. Risk Anal 1995; 15: 369-390.

46. Plans-Rubió P. Cost-effectiveness of cardiovascular prevention programs in Spain. Int J Technol Assess Health Care 1998; 14: $320-330$.

47. Montserrat JM, Amilibia J, Barbé F, et al. Tratamiento del síndrome de las apneas-hipopneas durante el sueño. Arch Bronconeumol 1998; 34: 204-206.

48. Barbé F, Mayoralas L, Durán J, et al. Treatment with continuous positive airway pressure is not effective in patients with sleep apnea without daytime sleepiness. A randomised controlled trial. Ann Intern Med 2001; 134: $1015-1023$.
49. Stradling JR. Obstructive sleep apnoea: definitions, epidemiology, and natural history. Thorax 1995; 50: 683-689.

50. Peppard PE, Young T, Palta M, Dempsey J, Skatrud J. Longitudinal study of moderate weight change and sleep disordered breathing. JAMA 2000; 284: 3015-3021.

51. Newman AB, Nieto FJ, Guidry U, et al. Relation of sleepdisordered breathing to cardiovascular disease risk factors: the Sleep Heart Health Study. Am J Epidemiol 2001; 154: 50-59.

52. McNicholas WT. Obstructive sleep apnea syndrome: who should be treated? Sleep 2000; 23: Suppl. 4, S187-S190.

53. Brazier J, Deverill M, Green C, Harper R, Booth A. A review of the use of health status measures in economic evaluation. Health Technol Assess 1999; 3: 83-95. 